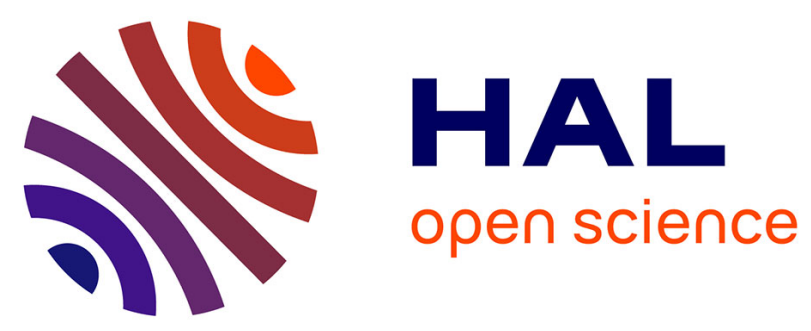

\title{
Genre et accord dans quelques dialectes arabes
}

Dominique Caubet, Marie-Claude Simeone-Senelle, Martine Vanhove

\section{To cite this version:}

Dominique Caubet, Marie-Claude Simeone-Senelle, Martine Vanhove. Genre et accord dans quelques dialectes arabes. LINX 21: Genre et langage. Actes du Colloque tenu à Paris X - Nanterre les 14-15-16 décembre 1988, Mar 1989, France. pp.39-66. halshs-00009753

\section{HAL Id: halshs-00009753 https://shs.hal.science/halshs-00009753}

Submitted on 24 Mar 2006

HAL is a multi-disciplinary open access archive for the deposit and dissemination of scientific research documents, whether they are published or not. The documents may come from teaching and research institutions in France or abroad, or from public or private research centers.
L'archive ouverte pluridisciplinaire HAL, est destinée au dépôt et à la diffusion de documents scientifiques de niveau recherche, publiés ou non, émanant des établissements d'enseignement et de recherche français ou étrangers, des laboratoires publics ou privés. 
Dominique CAUBET, Université de Besançon, CNRS, U.A.1066, Paris III, U.A. 1028, Paris 7 ,

Mar1e-Claude SIMEONE-SENELLE, CNRS, U.A.1066, Paris III,

Martine VANHOVE, U.A.1066, Paris III.

GENRE ET ACCORD DANS QUELQUES DIALECTES ARABES

I - IE GENRE

1 - Le genre en sémitique

Historiquement, en sémitique, il semble que le genre n'était pas marqué dans la morphologie des substantifs, et qu'il ne se manifestait que dans les phénomènes d'accord, en particulier avec les pronoms.

Les langues sémitiques possèdent peu de suffixes puisqu'elles utilisent essentiellement le procédé de dérivation par schèmes pour la création de mots. Cependant, on note l'apparition d'un suffixe $-(a)(t)$, d'origine vraisemblablement déictique, qui permet, dès le départ, de marquer certaines oppositions :

- il permet d'opposer un singulatif au collectif pour les nonhumains,

- un groupe humain à un individu,

- un sens spéclalisé à un sens propre,

- 11 permet la création de noms abstraits.

David Cohen explique que ce n'est que plus tardivement, et "par raccroc", que ce suffixe sera amené à marquer l'opposition masculinféminin, la classe des féminins constituant la classe marquée.

En ce qui concerne les verbes et les pronoms, en arabe classique, il existe une opposition de genre marquée morphologiquement aux 2ème et 3 ème pers. du singulier, duel et pluriel.

2 - Etat actuel dans quelques dialectes arabes

Les dialectes étudiés pour cet article concernent les régions suivantes :

Mauritanie : dialecte hassānīya,

Maroc : dialecte des environs de Fès,

Algérie : Alger,

Malte : maltais standard et dialectal,

Tunisie : dialectes des Marazig, de Takrouna, de Al-Hamma de Gabès, de Sfax et du Kef,

Egypte : arabe médian ("educated") du Caire,

Liban : dialectes de la montagne et des grandes villes du littoral,

Syrie : Damas,

Yémen du Nord : dialectes de Đ̣afār, al-Mahall, im-Mał̣țah, Șanăă Sakdah...

(cf. bibliographie pour les références précises) 
Il existe deux types de marqueurs différents pour souligner l'appartenance au féminin, selon qu'il s'agit des pronoms et des verbes d'une part, et des nominaux (substantifs, adjectifs, participes) d'autre part.

\section{a - Les verbes}

Dans les verbes, les dialectes arabes peuvent connaitre des distinctions de genre pour certaines personnes et à certaines conjugaisons. Rappelons qu'il n'y a que deux formes verbales : la conjugaison suffixale (valeur d' "accompli"), et la conjugaison préfixale (valeur d" inaccompli").

Il existe des degrés dans la façon dont les distinctions de genre sont rendues dans les dialectes arabes ; certains d'entre eux connaissent une distinction "maximale", qui oppose le féminin au masculin aux 2 ème et 3ème personnes du singulier et du pluriel ; d'autres, par contre, ne marquent cette distinction qu'au singulier, et seulement pour la 3ème personne (maltais, marocain pour la conjugaison préfixale...).

\section{1 - la conjugaison suffixale}

La conjugaison suffixale, comme son nom l'indique, est constituée au moyen d'indices de personne post-posés au radical du verbe.

- Pour les dialectes qui ont maintenu la distinction maximale, nous citerons l'exemple du verbe qui signifie "écrire" (1) :

$$
\text { Ar.class. }
$$

SG. 1 .

.m. katab-to

2.f. katab-ti

3.m. katab

3.f. katab-at

PL. 1

2.m. katab-tum

2.f. katab-tunna

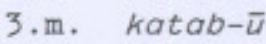

3.f. katab-na

\section{Tunisie}

(Marazig)

$k t \partial b-t$

$k t \partial b-t$

$k t \partial b-t i$

$k t \curvearrowright b$

$k a t b-a t$

$k t ə b-n a$

$k t a b t-u$

$k t \partial b-t a n$

$k a t b-u$

$k a t b-a n$
Nord-Yémen

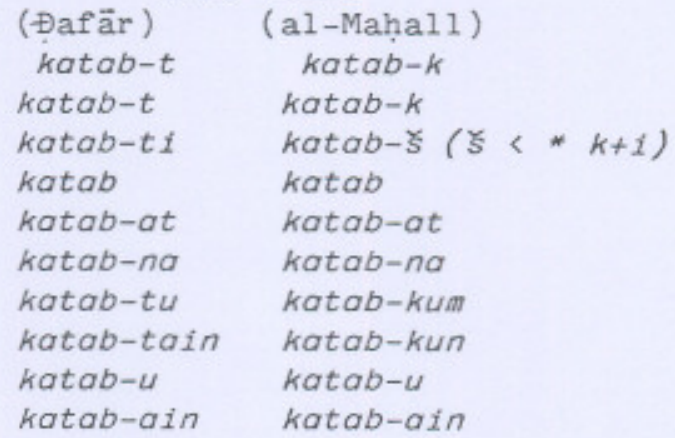

Beaucoup des dialectes modernes étudiés ici n'ont maintenu la distinction de genre qu'au singulier, à la 2ème ou à la 3ème personne:

- A la 2ème personne, certains dialectes distinguent un masculin en $-t$ (identique à la lère personne), d'un féminin en $-t \underline{1}$ (Tunisie (al-Hämma, Sfax, le Kef, Mauritanie...) : 


\begin{tabular}{|c|c|c|c|}
\hline \multirow{4}{*}{ SG. } & & $\begin{array}{l}\text { Tunisie } \\
(\operatorname{Sfax} . . .)\end{array}$ & Maur $1 \tan 1$ \\
\hline & 1. & $k t \curvearrowright b-t$ & $k t \curvearrowright b-t$ \\
\hline & $2, \mathrm{~m}$. & $k t \ominus b-t$ & $k t \ominus b-t$ \\
\hline & 2. $f$. & $k t a b-t i$ & $k t \partial b-t i$ \\
\hline \multirow{5}{*}{ PL. } & $3 . \mathrm{m}$. & $k t \curvearrowright b$ & $k t \partial b$ \\
\hline & 3.1. & $k \partial t b-\partial t$ & $k \partial t b-\partial t$ \\
\hline & 1. & $k t \partial b-n a$ & $k t \curvearrowright b-n a$ \\
\hline & 2. & $k t \partial b-t u$ & $k t \partial b-t u$ \\
\hline & 3. & $k a t b-u$ & $k \partial t b-u$ \\
\hline
\end{tabular}

- La distinction minimale est celle qui oppose une 3ème personne du masculin, avec une marque $\emptyset$, à une zème personne du féminin suffixée en $-t$ :

\begin{tabular}{|c|c|c|c|}
\hline \multirow{3}{*}{ SG. } & & Maroc & Malte \\
\hline & 1. & $k t \curvearrowright b-t$ & $k t i b-t$ \\
\hline & 2. & $k t a b-t i$ & $k t i b-t$ \\
\hline \multirow{5}{*}{ PL. } & $3 . \mathrm{m}$. & $k t a b$ & $k i t e b$ \\
\hline & 3.1. & $k \partial t b-\partial t$ & $k i t b-e t$ \\
\hline & 1. & $k t \partial b-n a$ & $k t i b-n a$ \\
\hline & 2. & $k t a b-t u$ & $k t i b-t u$ \\
\hline & 3. & $k \partial t b-u$ & $k \perp t b-u$ \\
\hline
\end{tabular}

Remarquons que l'arabe marocain qui entre dans ce type d'opposition minimale pour le genre, a la particularité d'avoir conservé une forme en -1 pour la 2 ème personne, marquant ainsi une opposition de personne (2ème:1ère), et non de genre. Par contre, le maltais ne fait pas d'opposition entre la lère et la 2ème personne du singulier.

\section{2 - la conjugaison préfixale}

La conjugaison préfixale contient un indice de personne préfixé et un indice de nombre suffixé (sauf à la lère personne dans quelques dialectes : sg. Jaktub, pl. noktub); dans certains dialectes (marocain, tunisien de Sfax, des Marazig, hassānĪya...), elle peut également porter une marque de genre suffixée à la 2ème pers. du singulier. Pour tous les dialectes, $11 \mathrm{y}$ a opposition de genre à la 3ème personne du singulier, sous forme d'un indice préfixé ( $t-$, pour le féminin, $y-$, pour le masculin).

- Comme pour la conjugaison suffixale, on trouve des dialectes qui ont une opposition de genre au pluriel, ce qui représente le degré maximal :

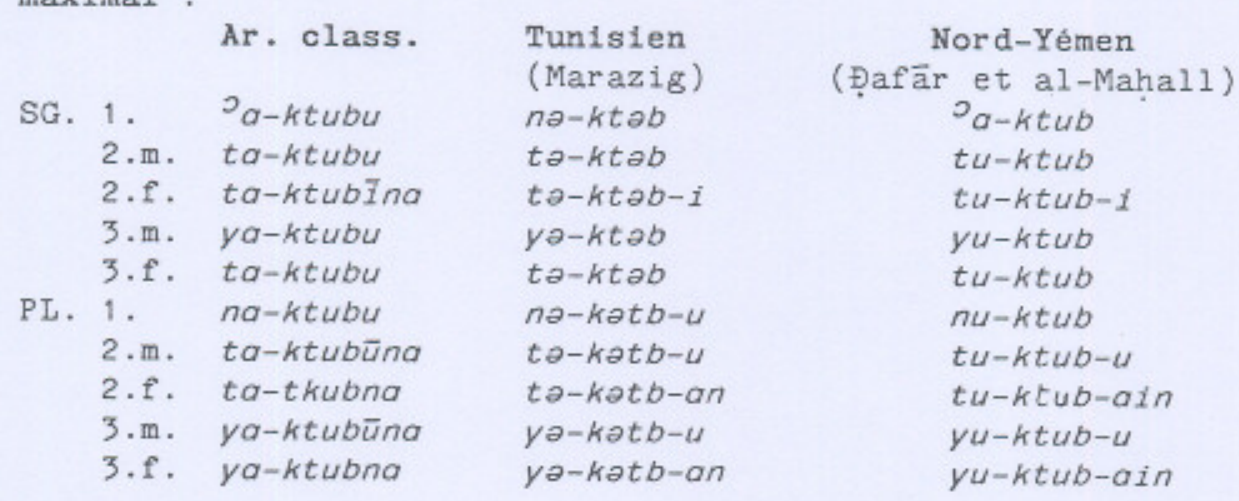




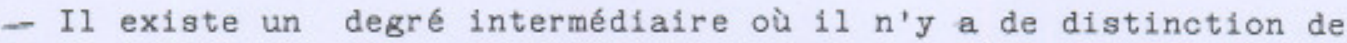
genre qu'aux 2ème et 3 ème personnes du singulier (toutes les formes du pluriel sont suffixées en $-\mu$, quel que soit le genre):

SG. 1

\section{marocain}

$n \curvearrowright-k t \partial b$

$2 . \mathrm{m} . \quad t 2-k t \partial b$

2.f. $t-k a t b-1$

3.m. $y ə-k t \partial b$

3.f. $t 2-k t \partial b$

PL. 1. nə-kətb-u

2. $t-k a t b-u$

3. ya-kətb-u

$$
\begin{aligned}
& \text { HassänIya } \\
& \text { nə-ktəb } \\
& t \partial-k t \partial b \\
& t \partial-k t \partial b-i \\
& y \partial-k t \partial b \\
& t \partial-k t \partial b \\
& n a-k t b-u \\
& t \partial-k t b-u \\
& y \partial-k t b-u
\end{aligned}
$$

- Enfin, le maltais présente le degré minimal, puisqu'il n'oppose les genres qu'à la 3ème personne du singulier :

$$
\begin{aligned}
& \text { SG. 1. ni-kteb } \\
& \text { 2. } t i-k t e b \\
& \text { 3.m. } \quad y i-k t e b \\
& \text { 3.f. } t 1-k t e b \\
& \text { PL. 1. } n i-k t b-u \\
& \text { 2. } t i-k t b . u \\
& \text { 3. } y i-k t b-u
\end{aligned}
$$

Il faut remarquer que l'arabe marocain n'offre pas de symétrie dans la distinction de genre dans les deux conjugaisons (c.suff., seulement à la 3ème pers. ; c.préf., 2ème et 3ème pers, du singulier).

b - Les pronoms personnels indépendants

$$
1 \text { - Le singulier }
$$

Dans la grande majorité des cas, il n'y a qu'un seul pronom pour la 1 ère personne ; des dialectes du Nord-Yémen ont des pronoms différenciés à la lère personne.

En outre, on remarque que dans le dialecte de im-Mattah, au Yémen du Nord, la 3ème personne est indifférenciée, contrairement à ce qui se passe dans les autres dialectes.

Ar.cl.

SG. 1.m. ${ }^{2} g n \bar{a}$

1. f.

2.m. Panta

2.f. Tanti

3.m. huwa

3.f. hiyo

2 - Le pluriel

\section{Nord-Yémen Đafär im-Ma丰ah}

?ana 'anā

$\mathrm{o}_{a n i}$

onta

ont 1

hü

$h \bar{i}$

onta

Janti

${ }^{2} a h \bar{a}$

วahā
Tunisie

Maraz. Takroun.

äne äna äna

\section{enta}

hü hu

hĩya hïya

Maur. Malte Maroc

äna $\quad y \overline{i \partial n}(a) \bar{a} n a$

antä int( $i)$ anto

anti int( $i)$ anti

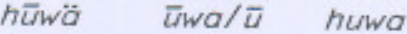

hĩyä $\bar{i} y a / \bar{i}$ hiya

Au pluriel, on peut discerner deux types de dialectes : ceux qui ont une distinction de genre (seulement pour les 2ème et 3ème personnes), avec un degré maximal d'opposition; et ceux qui ne distinguent pas le genre au pluriel, qui représentent le degré minimum : 


\begin{tabular}{|c|c|c|c|c|c|c|c|}
\hline \multirow{6}{*}{ PL. } & \multirow{2}{*}{ Ar.cl. } & Nord-Yémen & Tun1si & \multirow{2}{*}{ Takroun } & \multirow{2}{*}{ Maur. } & \multirow[t]{2}{*}{ Malte } & \multirow[t]{2}{*}{ Maroc } \\
\hline & & Đ̦afär im-Mațțah & Maraz & & & & \\
\hline & nahnu & nithriā & & (n)ehnnä & $(n) h a n a ̈$ & ahna & \\
\hline & $\begin{array}{l}2 . \mathrm{m} \cdot{ }^{2} \text { antum } \\
2 . \mathrm{f} \text {. antunna }\end{array}$ & $\begin{array}{l}\text { antu }(m) \text { antum } \\
\text { antain antinna }\end{array}$ & entum & entum & əntūmä & Intom & entūma \\
\hline & 3.m. hum & hum $\partial_{\text {ihim }}$ & hum & hum & hūmä́ & $\bar{u} m a$ & hüma \\
\hline & 3.f. hunna & hin(han) o ihinno & hen & hum & $h \bar{u} m \ddot{a} t \dot{i}$ & $\bar{u} m a$ & $n \bar{u} m a$ \\
\hline
\end{tabular}

\section{c - Les nominaux}

Nous envisagerons successivement les cas des substantifs et ceux des autres nominaux (adjectifs ou participes).

\section{1 - Les substantifs}

Les substantifs se distinguent des autres nominaux en ce qu'ils ont un genre qui leur est propre. On remarque aujourd'hui dans les dialectes arabes que la très grande majorité des substantifs féminins sont porteurs d'un suffixe $-a(t)$. Le suffixe apparait, selon les dialectes, sous une forme $-a(t),-\ddot{a}(h),-e(h) \ldots$

Cependant de nombreux phénomènes particuliers permettent de remettre en cause l'équation : suffixe $-\sigma(t)=$ féminin.

\section{a - Existence de substantifs féminins sans marque}

Il existe un certain nombre de substantifs de genre féminin (comme on peut l'observer dans l'accord avec les verbes, les adjectifs ou les pronoms) qui ne portent pas de morphème de genre ; 11 est intéressant de noter qu'il s'agit bien souvent des mêmes lexèmes limités à certains champs sémantiques (on les retrouve également en arabe classique):

- les parties du corps (en particulier les parties doubles),

- des noms d'objets inanimés (instruments, vêtements, armes...),

- des noms de lieux (maison, marché, route...),

- des noms d'éléments naturels,

- des noms d'aliments (beurre, huile, miel...),

- des noms d'êtres animés de sexe féminin,

- des noms de villes,

- des noms de langues.

On constate dans les dialectes une amorce de normalisation du système : certains noms féminins non marqués morphologiquement ont tendance soit à devenir masculins soit, au contraire, à prendre une marque $-a(t)$.

Il est également intéressant de noter qu'il existe une tendance inverse : certains termes des catégories sémantiques ci-dessus, masculins en arabe classique, sont féminins dans des dialectes, parfois sans changement morphologique. Peut-etre est-ce par analogie avec les autres termes de la catégorie?

1 - Sont féminins sans marque dans tous les dialectes (comme en arabe classique) des parties du corps et des éléments naturels : oeil, main/bras, âme/souffle,

terre et soleil.

Signalons en outre, pour certains dialectes :

- des noms d'êtres animés de sexe féminin :

- Maroc : xādəm servante 


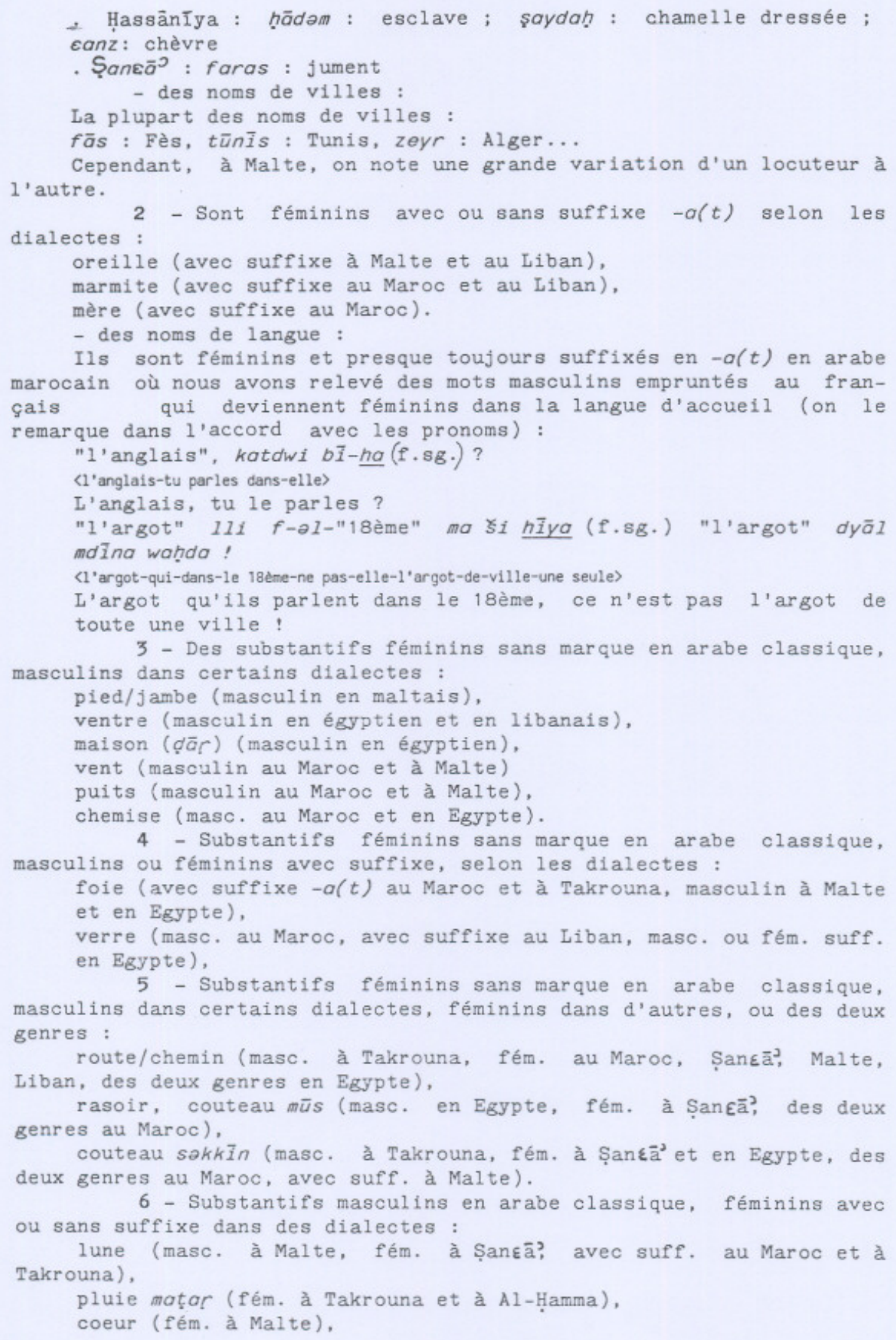


miel et huile (fém. au Maroc),

b - Les masculins en $-a$

Cette terminaison $-a$ peut avoir des origines très différentes :

$$
1 \text { - les substantifs masculins en }-a
$$

- Ce - $a$ final peut être issu d'une semi-consonne qui appartient. à la racine, et dont la longueur se neutralise en finale; c'est le cas de nombreux noms d'action :

$\epsilon m a$ : le fait d'être aveugle (masc. en marocain et égyptien, mais

fém. en maltais, sous la forme āma),

ğla : le fait d'être cher (marocain)....

- Il apparaît après la chute d'un $\varepsilon$ ou $\dot{g}$ à Malte, ou d'un 2 dans beaucoup de dialectes :

- Malte :

zara : grain ; raba : champ cultivé ; bama : boue ; sema : ciel

- Maroc : Liban)...

$m a$ : eau, dwa : médicament (ce terme est fém. à Malte et au

- Il s'agit d'emprunts désignant des êtres de sexe masculin :

- Malte:

mastrudał̧a : charpentier ; barba : oncle ; eremita : ermite

Cependant, on note, quelquefois, et en particulier en maltais qui ne subit plus aucune influence de l'arabe littéraire, une tendance à rendre féminins les noms ayant une terminaison $-a$, quelle que soit leur origine; peut-être par souci de normalisation. C'est ainsi que les jeunes citadins accordent zara (grain) et raba (champ cultivé) au féminin, alors qu'ils sont toujours au masculin chez les vieux paysans.

$$
2 \text { - les substantifs masculins en }-\sigma(t)
$$

- Au Yémen, Rossi donne toute une liste de substantifs masculins en $-e h$, pour Saneāo:

కaybeh : un vieux, rähīneh : un otage, bäకeh : un pacha, xalīfeh: un calife, earïfeh : un sergent.

- De même, au Maroc, on trouve :

$b \bar{a} \xi a$ : pacha, $x \not \bar{i} f a$ : calife.

Examinons maintenant les emplois du suffixe $-a(t)$.

c - Le suffixe $-a(t)$

Nous commencerons par examiner les différentes valeurs du suffixe, puis nous essayerons de voir s'il est possible de dégager un invariant derrière toutes ces valeurs.

1 - la diversité des emplois

Le suffixe $-a(t)$ remplit de nombreuses fonctions dans les dialectes arabes; nous avons relevé les emplois suivants :

- formation du nom de la femelle par opposition au mâle,

- formation du nom d'unité par opposition au collectif, 
- formation d'un substantif dénombrable à côté d'un indénombrable,

- formation de nom d'action

- formation du nom d'acte unique, pour le nom d'action,

- formation de pluriels (surtout pour les groupes humains),

- formation de certains collectifs (non-humains).

- formation d'emplois de spécification.

Nous donnerons quelques exemples pour chaque emploi dans un certain nombre de dialectes.

\section{1 - formation du nom de la femelle}

Il s'agit généralement d'opposer la femelle au mâle ou la femme à 1 'homme (noms de métier...) ;

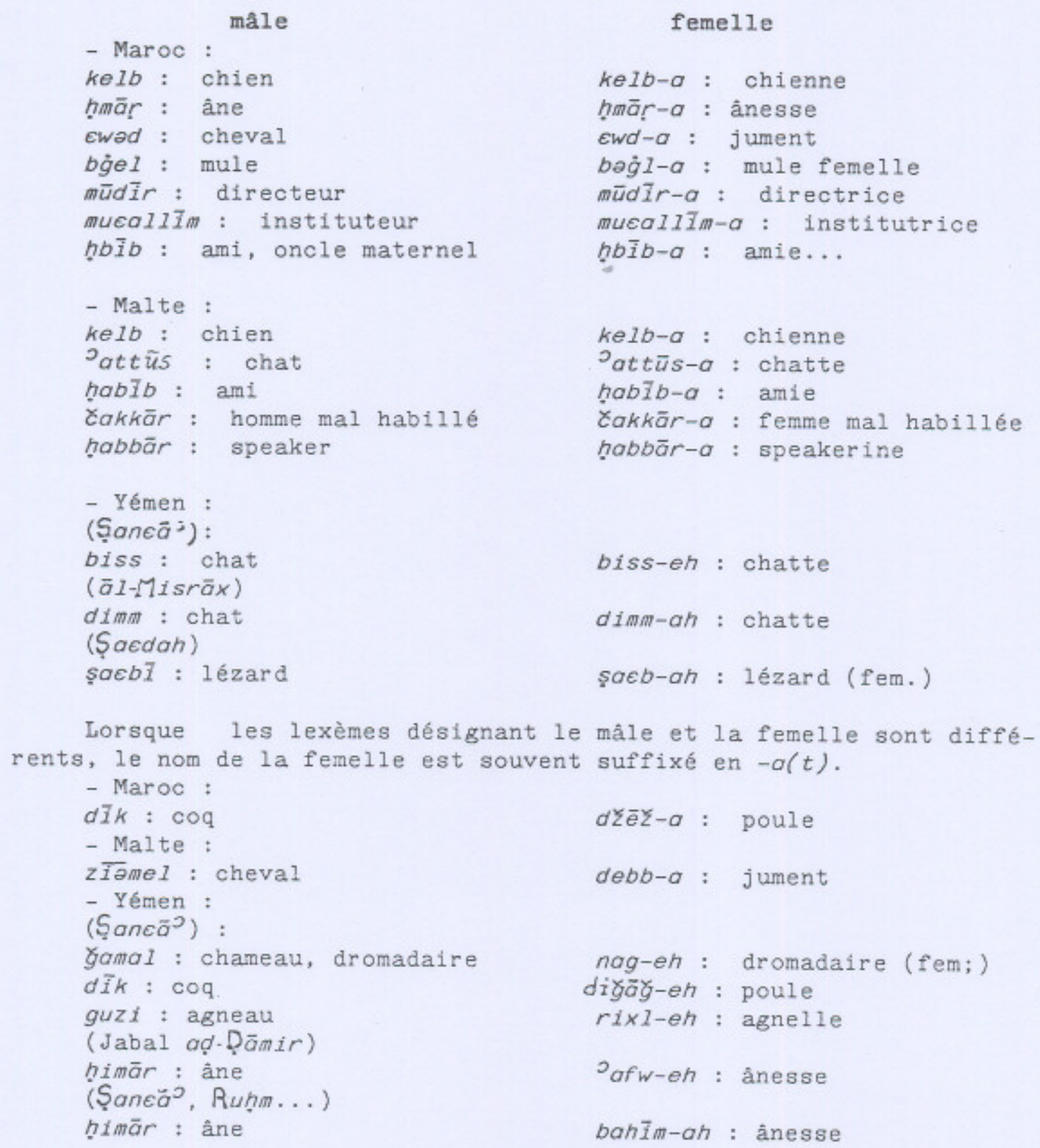




\section{2 - formation du nom d'unité de collectifs}

Le suffixe $-a(t)$ sert également à former le nom d'unité pour les substantifs collectif's; les collectif's désignent une classe formée d'éléments pris en bloc, sans qu'il y ait individuation ; $11 \mathrm{~s}$ concernent surtout le domaine sémantique des petits animaux (ou des animaux considérés en troupeaux), de la nourriture (fruits et légumes, oeufs...).

Le suffixe $-a(t)$ permet de constituer un substantif désignant un individu de la collection; il s'agit d'un passage de la catégorie du continu (indénombrable) à celle du discontinu (dénombrable). Nous prendrons quelques exemples pour chaque dialecte étudié.

Il faut remarquer que cette formation nominale, qui permet le passage à la catégorie du discontinu, peut se pluraliser en -āt ; ce pluriel est souvent appelé "pluriel de paucité" parce qu'il s'utilise pour les constructions avec les numéraux inférieurs à 12, et qu'employé seul, il permet de désigner un petit nombre d'individus (emploi que l'on pourrait rendre par le "quelques" français).

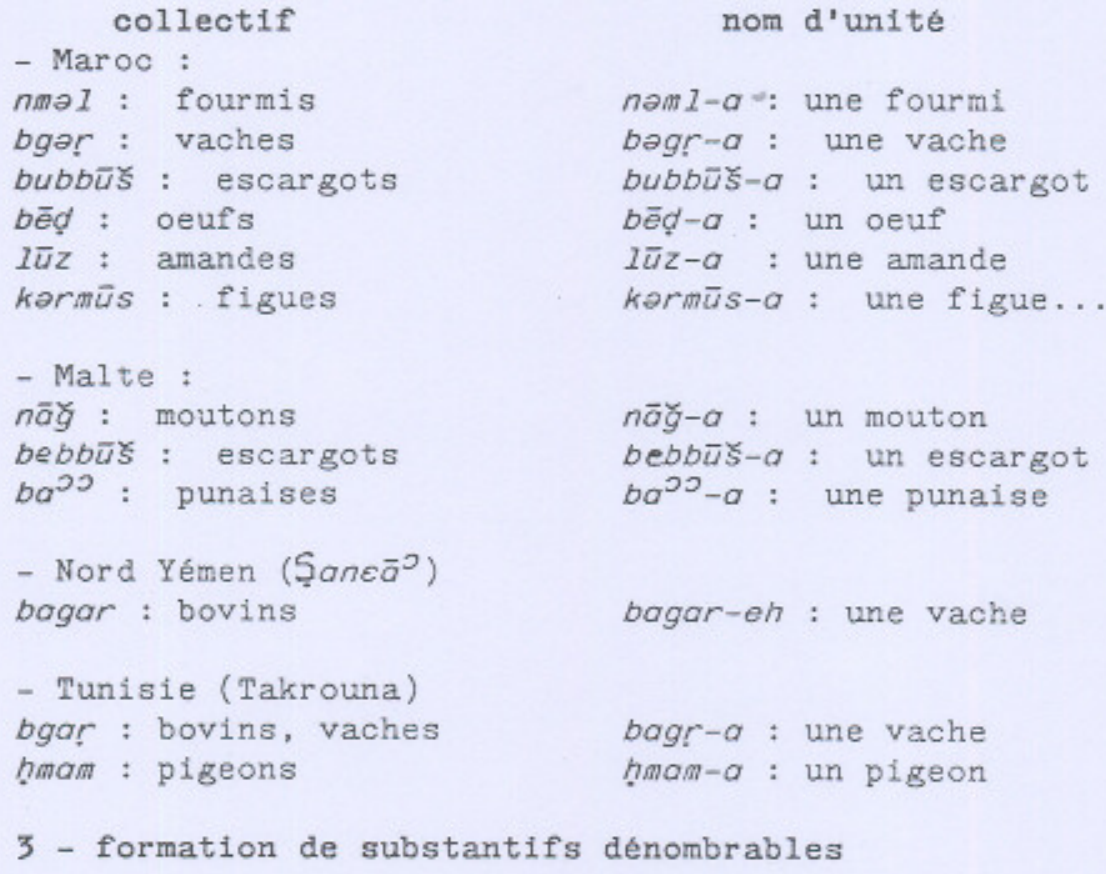

Nous avons relevé un procédé de passage de la catégorie du continu (indénombrable) à celle du discontinu (dénombrable) qui implique le suffixe $-a(t)$; dans certains dialectes, la simple suffixation suffit. D. Cohen parle de "nom de portion".

$$
\begin{aligned}
& \text { - Malte: } \\
& \text { hobz: pain } \\
& \text { bzōr : poivre }
\end{aligned}
$$

hobz-a: une miche de pain $b z a ̈ r-a$ : un grain de poivre 
- Tunisie(Takrouna):

esal : miel

ḥuøș : pois chiches

lhäm: viande, chair casl-a: une goutte de miel

(en poésie)

humşa : un pois chiche

lahma : un morceau de viande

Il s'agit d'un phénomène lié à la formation du nom d'unité pour les collectifs, puisque l'on désigne une quantité dénombrable.

En arabe marocain, le suffixe doit être associé à un schème de diminutif ; les substantifs ainsi formés se comportent comme des discontinus, ce qui se ressent en particulier dans la détermination nominale :

substantif continu

esal : du miel

lbam : viande

tmar : dattes

sman : beurre conservé substantif discontinu

esill-a: un peu de miel

$1 h \overline{1} m-\sigma$ : un peu de viande

$t m \bar{i} r-a$ : un peu de dattes

$s m \bar{i} n-a$ : un pea de beurre conservé

\section{4 - formation de noms d'action}

Le suffixe $-a(t)$ est associé à certains schèmes de noms d'action:

verbe

- Maroc :

qnat : s'ennuyer

kahh : tousser

gāor : être jaloux

$z \varepsilon \bar{a} m$ : être courageux noms d'action

$q \partial n t-a$ : fait de s'ennuyer

$k ə h h-\sigma$ : fait de tousser

$\dot{g} \bar{e} r-a$ : fait d'être jaloux

zeăm-a : fait d'être courageux...

5 - formation d'un nom d'acte unique

$-a(t)$ suffixé à un nom d'action permet de distinguer entre le nom d'action abstrait (sans suffixe), et l'acte unique (D. Cohen parle de "nom d'opération") :

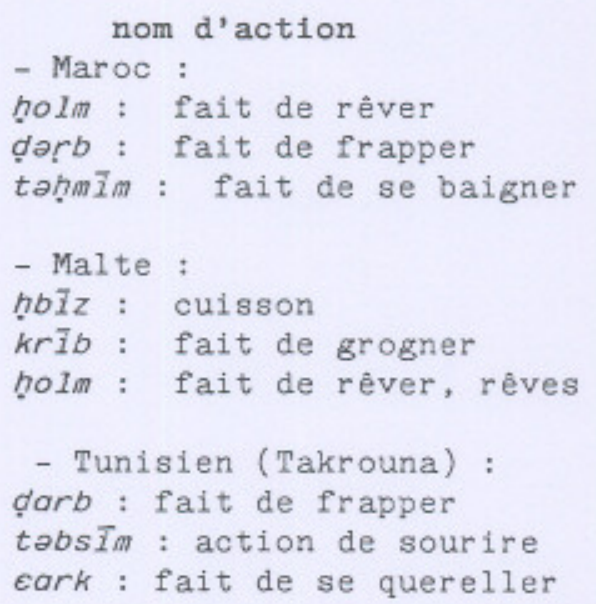

Mais la suffixation de $-a(t)$ peut aussi conférer à un nom d'action la valeur de terme générique (Takrouna) : 
đ̣arba : "frappement, façon de frapper (nom modal)", par opposition à garb : "fait de frapper".

\section{6 - formation de pluriels}

Le suffixe $-a(t)$ entre dans la formation de certains pluriels ; il s'agit généralement des pluriels de groupes humains (noms de métier, ethnonymes...) ; ce suffixe servait à former des collectifs humains dans les langues sémitiques; dans les dialectes arabes, ce sont des pluriels. Pour les ethnonymes, on a parfois modification du

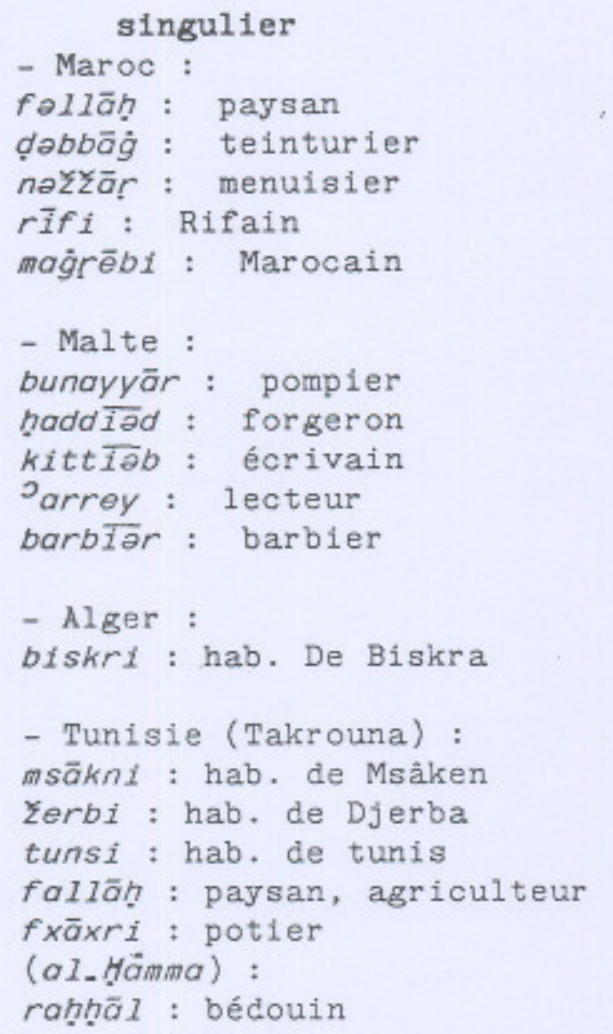

\section{pluriel}

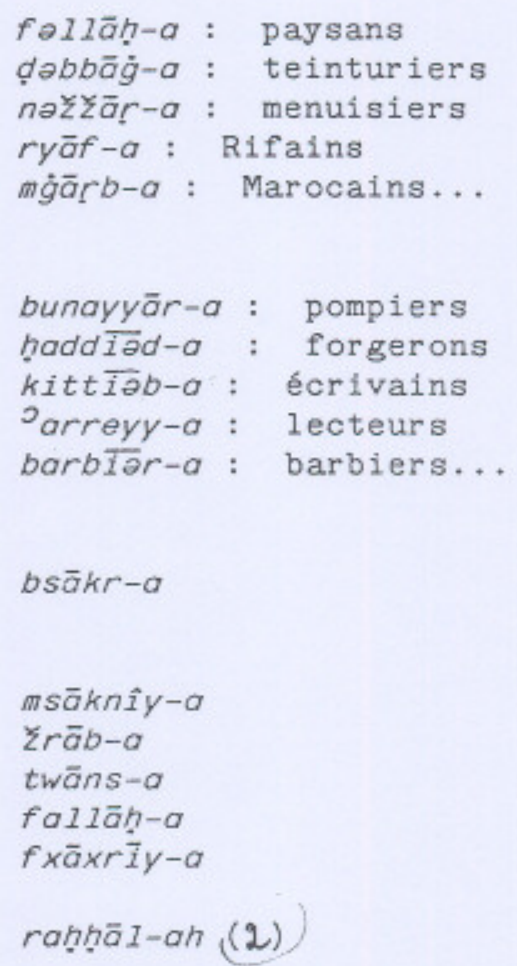

- A Saneā?, au Yémen, on trouve des pluriels de groupes d'animaux (le singulier est en -1 ) :

Dans certains dialectes, des collectifs inanimés ont une terminaison $-a(t)$ (n'ayant pas toujours pour origine le suffixe $-a(t)$ ). - Malte :

zeba : teintures, āmāra : meubles, fazōla: haricots

(2) Ces pluriels en $-a(t)$ ont aussi souvent un pluriel en $\bar{i} n$ : Takrouna : zauwārīn et zauwāra: falsificateurs, zahwānīn et zahwānīya: hommes de caractère gai ; Malte : rahhâla et rahhalinn : villageois. 
- au Maroc, ils sont très nombreux :

māțēsa : tomates, lūbya : haricots, xūdra: légumes, fəlf la : poivrons, gərea : courgettes, bəșla : oignons, そəlbāna : petits-pois, halwa : gâteaux (3).

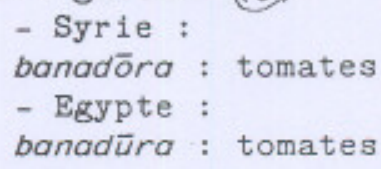

Derrière cette longue liste d'emplois différents, peut-on essayer de dégager un invariant?

11 - à la recherche d'un invariant

Nous commencerons par rappeler le rôle de ce suffixe dans les langues sémitiques, puis nous essayerons de chercher une opération commune à tous ces emplois dans les dialectes arabes modernes.

1 - rappel historique

David Cohen pose l'hypothèse que ce marqueur est au départ un article de type déictique; en tant que suffixe, sa fonction la plus anciennement attestée est double :

- une marque de collectif pour les humains,

- un singulatif pour les collectifs non-humains.

Il signale d'autres emplois dans la formation des noms abstraits, la nominalisation des adjectifs et dans des emplois métaphoriques.

David Cohen, évoquant la pauvreté des langues sémitiques en (3) Le nom d'unité est souvent semblable au collectif ; ainsi, on trouve en arabe marocain : māțēsa : une tomate, falf la : un poivron, gərca : une courgette, bəșla : un oignon, Zalbāna : un petit-pois (pas encore écossé), halwa : un gâteau ; par contre, on utilise un classi-

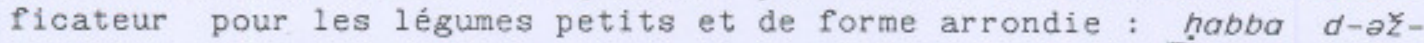
žlbāna : un petit-pois (grain); habba d-al-lübya : un haricotblanc. 
suffixes, explique qu'ils ont dû servir à l'équilibration formelle des systèmes.

Il attribue à ce suffixe un rôle d'actualiseur, de spécificateur, en tant qu'ancien élément déictique, porteur de détermination. $-a(t)$, dont la vacuité sémantique a permis cette "omnivalence" n'est cependant pas dépourvu de fonction.

\section{2 - Peut-on dégager un Invariant dans les dialectes actuels ?}

Nous allons essayer de regrouper les huit emplois que nous avons relevés dans les dialectes.

Tout d'abord, nous avons noté des valeurs qui permettent de passer d'une catégorie à l'autre : de la catégorie du continu (indénombrable, appréhendé de façon globale), à celle du discontinu (décomposable en unités).

\section{1 - Le passage du continu au discontinu}

Un certain nombre d'emplois peuvent se regrouper sous cette opération :

- la formation du nom d'unité (discontinu) pour les collectifs (continus),

- l'opposition entre l'acte unique (discontinu) et le nom verbal abstrait (continu),

- le passage à la catégorie du discontinu, parfois en association avec un schème de diminutif, pour les substantif's continus désignant la nourriture en particulier.

Tous ces emplois peuvent s'analyser en termes de recatégorisation : passage du continu au discontinu.

\section{2 - Le passage au continu, le lissage}

On a également observé le phénomène inverse : la suffixation en $-a(t)$ permet d'envisager des groupes humains en bloc, et non plus comme une somme d'individus ; 11 s'agit d'une opération de lissage, où toutes les différences sont gommées, et où on appréhende le groupe comme un ensemble continu.

Il en est de même pour la formation des noms d'action, qui permettent de faire référence directement à des propriétés ("le fait de..."), sans passer par un dénombrement.

\section{3 - 1'opposition masculin-féminin}

Le suffixe permet aussi de marquer des oppositions entre une classe marquée morphologiquement et une classe non marquée morphologiquement.

Nous regrouperons toutes ces opérations sous une opération plus générale de construction d'une classe, de "délimitation", qu'il s'agisse de la construction d'une classe d'occurrences associée à une notion (un ensemble de propriétés), pour les deux premières opérations de recatégorisation (passage du discontinu au continu, ou du continu au discontinu), ou de la construction d'une classe marquée, par 
opposition à une classe non marquée.

Avant d'aborder le problème spécifique de l'accord, nous allons donner un bref aperçu de l'opposition de genre dans les adjectifs et les participes.

\section{d - les adjectifs et les participes}

- Les adjectifs peuvent avoir trois ou quatre formes différentes :

- deux formes de singulier, dont une suffixée en $-a(t)$,

- une ou deux formes de pluriel.

Dans certains dialectes, les adjectifs $\in n-1$, qui forment un pluriel externe en $-\bar{i} n$, ont souvent une forme de féminin pluriel en $-\bar{a} t$.

L'existence de ces formes, que nous allons étudier brièvement, ne préjuge pas d'une automaticité de l'accord ; c'est ce que nous verrons dans la dernière partie.

- Dans certains des dialectes étudiés ici, il y a des adjectifs qui n'ont qu'une forme de singulier ; on distingue plusieurs raisons à cette invariabilité.

\section{1 - Les adjectifs sémantiquement féminins}

Ces adjectifs, comme en arabe classique, ne sont pas marqués morphologiquement comme féminins, mais ils ne peuvent s'appliquer qu'à des êtres de sexe féminin :

- Egypte :

bămil : enceinte, गōgir : infertile, ${ }_{a q} a \bar{l} m$ : stérile, walūd : fertile, $h \bar{a}^{2} 1$ : : pubère...

- Tunisie (Takrouna):

hāmil : enceinte (employé par les hommes)

cāger : stérile, inféconde, bālog̉ : pubère, adulte...

- Yémen (al-ljadā) :

hämil : enceinte

- Mauritanie :

maxōọ : près de mettre bas (chamelle); garzni : au lait peu abondant, mais fortifiant

- au Maroc, ces adjectifs sont suffixés en $-a(t)$ :

eagr-a : stérile, haml-a : enceinte...

2 - Les adjectifs invariables

a - les adjectifs en $-i$

- à Malte, il existe un certain nombre d'adjectifs en - $i$ invaria-

bles en genre et en nombre (il s'agit d'emprunts) :

attwāli : actuel, rāri : rare

- de même à Takrouna, quelques adjectifs de couleur :

hamri : roux, rougeâtre

ainsi que des ajectifs de relation s'accordant avec des substantifs non-humains (on remarque toutefois un flottement dans l'accord) :

bațāniya Zerbi : couverture de Djerba 
lebsa tünsi : façon de s'habiller tunisoise

gunnaya tūnsi/tūnsiya: chanson tunisoise

- adjectifs d'origine en Egyptien(cisallam) :

baladi "local", earabi "arabe", ng11zi "anglais...

b - Des adjectifs en $-a$ a Takrouna

$x u l q a:$ magnifique(s)

kəmnuSa: âpre(s), $\operatorname{dur}(e)(s)$ en affaire

c - Des adjectifs de couleur empruntés

Nous avons relevé des exemples au Maroc et à Malte.

- Malte : rōza : rose, viola : violet, kannella : marron

- Maroc : rōz : rose, grūna : grenat, bordeaux, mōv : mauve

d - Certains adjectifs en arabe égyptien "éduqué"

- adjectifs de qualité sur le schème CaCūC :

laeūb "joueur", haqūd "méprisable", gayūr "jaloux"...

- adjectifs à finale $-\bar{a}^{\supset}$ :

şamma $\bar{a}^{\supset}$ "sourd", hayf $\bar{a}^{\supset}$ "mince", కamț $\bar{a}^{\supset}$ "grisonnant"...

e - Les élatifs

Enfin, on remarquera que les élatifs sont invariables :

- Takrouna :

așgar : plus petit, akbar : plus grand, âgé

- Maroc:

kbər : plus grand, qəll : moins, șgor : plus petit

āmina kbar man $x \bar{u}-k$ : Amina est plus vieille que ton frère. 〈amina-plus grand-frère-kf(b-toi〉

La comparaison peut aussi se faire au moyen de l'adjectif, avec une préposition différente ; dans ce cas, l'adjectif s'accorde en genre ou en nombre :

ämīna kbïra $\varepsilon l a x \bar{u}-k$ : Amina est plus vieille que ton frère.

〈amina-grande-sur-frere-toi〉

II - L'ACCORD

Nous examinerons successivement la question de l'accord de l'adjectif (et du participe) avec le substantif féminin singulier, pluriel, l'accord du verbe avec le sujet, et nous consacrerons un chapitre particulier aux collectifs.

1 - L'accord de l'adjectif ou participe avec le substantif

a - Le substantif est féminin singulier

- A I'exception des adjectifs invariables en genre et/ou nombre (cf.I d.2 ) I'accord est marqué au féminin par l'adjonction d'un suffixe $-a(t)$.

Cet accord est d'ailleurs un des signes qui permet de reconnaitre des substantifs féminins sans marque, puisque l'adjectif $s$ 'accorde au féminin, ainsi que la forme verbale et les pronoms anaphoriques. 


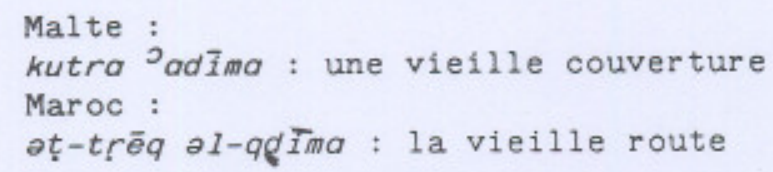

b - Le substantif est au pluriel féminin ou masculin

1 - L'adjectif est au pluriel

Il est rare que les dialectes fassent une distinction de genre au pluriel ; ceci ne concerne que les adjectifs qui ont une forme de pluriel externe (4)); dans ce cas, ils opposent un pluriel masculin en $-\bar{i} n$, à un pluriel féminin en $-\bar{a} t$. Pour les adjectifs qui ont un pluriel interne, il n'y a qu'une seule forme de pluriel, la différence de genre ne pouvant se marquer que par suffixation.

L'accord au féminin pluriel en -āt pour les adjectifs semble réservé à trois cas :

- pour des adjectifs d'appartenance à un groupe social (dont on ne sait pas s'ils sont vraiment adjectifs ou substantifs), quand ils sont associés à des substantifs désignant des humains ou gros animaux domestiques de sexe féminin :

- Syrie (Damas) : (accord au masc. pl. ou fém. pl.)

nəswān masalmīn/masəlmāt : des femmes musulmanes

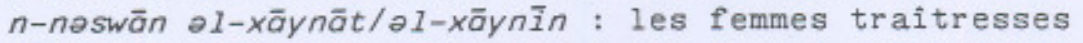

On trouve ce même genre de phénomène au duel :

al-bantēn al-halwīn/al-halwät : les deux belles filles

- Takrouna :

zəbbād (masc.), zəbbāda (fém.) : "qui donne beaucoup de beurre (lait, bête; plante, paturage dont la consommation favorise la production de beurre)". Lorsque le substantif est inanimé, un seul pluriel : zabbādīn; lorsqu'il s'agit de bêtes laitières, le pluriel est marqué comme féminin : zabbādāt.

Il $y$ a parfois flottement dans l'accord pour quelques rares adjectifs qui ne peuvent s'appliquer qu'à des êtres humains :

$z \varepsilon \bar{l} m$ (masc.), $\quad z \varepsilon \bar{i} m a$ (fém.) : "habile, expert, qui a des qualités de chef, fém. maitresse femmen. Un pluriel commun : zeām et aussi un pluriel marqué masculin : $z \varepsilon \bar{I} m \overline{1} n$, et un pluriel marqué féminin : $z \in \overline{1} m a \tilde{t}$.

- Au Maroc, 11 semble que les formes de pluriel en -āt pour les substantifs entrainent souvent des adjectifs à pluriel externe en -āt ; il y aurait donc aussi un phénomène d'assonance :

hād-əl-ayālāt $\epsilon r \bar{b} b \bar{i} y a \bar{t}$ : ces femmes sont paysannes hād-əl-ayālāt ma \̧i earābīyāt : ces femmes ne sont pas arabes hād-ə1-bnāt fōsīyāt/fāsīy İn, bāợawīyāt, tūnsīyāt, fransōwīyāt... Ces filles sont de Fès, de Casa, de Tunis, françaises...

(4) L'arabe distingue deux types de pluriel : le pluriel interne formé sur des schèmes spécifiques, et le pluriel externe formé par la suffixation d'un morphème au substantif singulier. 
hād-ə1-bəgrāt rūmīyāt : ces vaches sont importées (originaire्لj de l'étranger) (5)

- d'autre part, au pluriel de noms d'unités de collectifs, formé $\$$ avec le suffixe -ãt, et utilisép avec les numéraux, ou pour désigner un petit nombre d'éléments :

Syrie (Damas) :

bānādōrayāt mowiyye/mōwiyyāt : des tomates juteuses partie.

Nous reparlerons du problème des collectif́s dans la dernière

A part ces quelques cas, l'accord se fait avec la forme unique de 1 'adjectif pluriel :

- Maroc :

ksōwi bōyəọ, $q \bar{o} q \bar{i} y \bar{i} n$ : des robes blanches, violettes

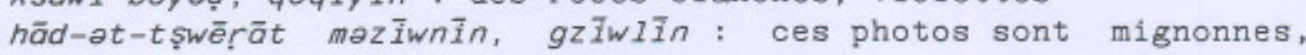
jolies

- Malte:

omos (fém. pl.) mahmuł̧īn : des chemises sales

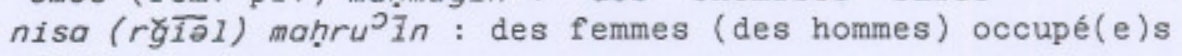

- Takrouna :

zəmnīn pluriel de zemni et de zamnïya : antique, ancien

\section{2 - L'adjectif est au "féminin singulier"}

- A Malte, avec les substantifs pluriels (humains ou non), on peut avoir l'accord de l'adjectif au féminin singulier (c'est rare, mais considéré comme idiomatique par les grammaires). C'est le cas dans les expressions figées : $b$ āyneyk (f.pl.) $m \bar{a} l \bar{u}^{\circ} a$ : les yeux fermés, ou en poésie : $\operatorname{toro}^{\circ}(\mathrm{f} . \mathrm{pl} \mathrm{f})$ tayba : de bons chemins.

- Pour certains dialectes (Maroc), nous serons amenées à poser d'emblée le problème des niveaux de langue; en effet, lorsque le sujet abordé est intellectuel au sens large, les dialectes non écrits sont souvent conduits àemprunter, soit à une langue étrangère comme le français, l'anglais, l'italien ou l'espagnol, soit à la langue littéraire.

En étudiant des corpus, on s'aperçoit que les emprunts lexicaux à l'arabe littéraire sont souvent assortis de règles d'accord particulières: pour les substantif's inanimés, l'arabe littéraire fait l'accord au féminin singulier et lors d'emprunts, cette règle est parfois respectée pour tous les éléments (verbe, adjectif, pronom), et parfois seulement pour une partie d'entre eux.

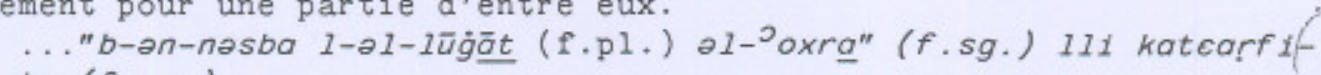
ha $(f . s g$.$) ...$

〈dans-le rapport-a-les langues-l'autre(f,)-que-tu connais-elle〉

... par rapport aux autres langues que tu connais...

bdāt (f.sg.) dāk-əd-duwwal (m.pl)... : Ces états ont commencé à - 〈elle a comencé-ce-les états〉 


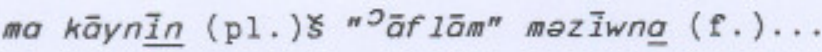

〈ne-il y a-pas-films-bonne〉

Il n'y a pas de bons films...

2 - L'accord du verbe avec le sujet

Dans les dialectes qui possèdent des formes verbales différenciées en genre au pluriel, l'accord se fait automatiquement : si le sujet est féminin pluriel, le verbe est au féminin pluriel, ni la place du sujet par rapport au verbe, ni la nature du sujet (animé ou inanimé) n'interviennent, contrairement aux règles d'accord en arabe classique.

A Malte, 11 est à noter que les grammaires signalent comme idiomatique un accord au féminin singulier avec un sujet au pluriel. Ce cas de figure ne s'est jamais présenté dans les corpus relevés sur le terrain.

\section{3 - L'accord avec les substantifs collectifs}

Les collectifs désignent traditionnellement des collections d'objets, d'animaux ou d'aliments, appréhendés en tant que groupes. Nous verrons qu'il existe également des substantifs désignant des groupes humains qui, bien que n'étant pas à proprement parler des collectifs, ont un comportement particulier pour l'accord.

\section{a - Les vrais collectifs}

Rappelons que ces substantifs désignent des groupes non-humains.

- L'accord avec le verbe ou les adjectifs se fait généralement au masculin singulier :

- Takrouna :

1s7l కahmu : sa graisse coule

〈il coule-graisse-lui〉

fär yaleab: des souris jouent

〈souris-il joue〉

- Maroc :

məzyān hād-əl-fūl! : elles sont bonnes, ces fèves : 〈bon-ce-le fève〉

- Malte :

wera ${ }^{2}$ nर्डšcf ya bad malayr

〈feuille-sèche-il prend feu〉

Les feuilles sèches prennent vite feu.

- Cependant, le collectif peut être ressenti comme un pluriel ; nous avons rencontré ce cas à Malte :

11-wera $\mathrm{ya}^{2} \mathrm{aw}$ mis-sigar : les feuilles tombent de l'arbre.

〈le feuille-ils tombent-de l'arbre〉

b - Les groupes humains

D'une manière assez générale dans les dialectes arabes, les substantifs désignant les groupes humains peuvent avoir un comporte- 
ment particulier à mi-chemin entre collectif et pluriel.

- Ces substantifs sont parfois des pluriels mais l'accord peut se faire au féminin singulier ou au pluriel :

- accord au f.sg. :

- Marazig

lowlad as-sğär txoḍd : les petits enfants courent en tous sens.

r-rammāya tsarrab: les lanceurs d'offrande défilent.

- Alger :

kānat an-nās $\varepsilon \bar{a}^{\supset} i \zeta a$ : les gens vivaient

〈elle était-les gens-vivante〉

ən-nās bdet tadxul 1-1ḥisb:les gens ont commencé à entrer au parti

〈les gens-elle a commence-elle entre-d-le parti〉

- Takrouna :

$m \xi \bar{a} t$ nās $u$ ¿āt nās : des gens sont partis et des gens sont venus

- Maroc :

an-nās ku1l-ha/kul1-hum : tous les gens

-les gens-tout-elle/eux>

- accord au pl. :

- Marazig :

yașarxu r-rammāya u yaḍhru: les lanceurs d'offrande tirent des coups de feu et sortent.

nnasawīn gālelli (gālan $+1 \bar{i})$ hekka : les femmes m'ont dit ceci.

- Takrouna :

ən-nsē yirkbu : les femmes montent à mule

- Alger :

on-nōs daxlu $w$ dōru : les gens sont entrés et lis ont fait

〈les gens-ils sont entres-et-ils ont fait〉

(cf. ci-dessus où l'accord est au féminin singulier chez le même locuteur)

- Yémen :

an-nās yuకullumuh : les gens le prennert

〈les gens-ils|prinnentlui〉

an-niswän yiglisēn yibkēn : les femmes s'asseoient en pleurant.

〈les femmes-elles s'asseoient-elles pleurent〉

- Au Maroc, nous avons trouvé dans un corpus, certains substantifs singuliers, désignant, de façon plus ou moins métaphorique, des groupes humains, repris par un pronom anaphorique au pluriel :

wähed-əd-dār (f.sg.), kāyn eand-hum (pl.)...

〈un la maison-11 y a-chez-eux〉

Dans une maison, ils ont....

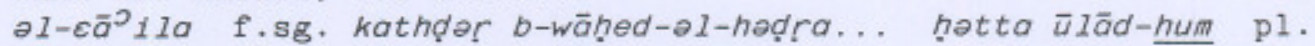
kaytebeü-hum pl.

〈la famille-elle parle-avec-un le parler-jusqu'a-fils-eux-ils suivent-eux〉

La famille parle un parler, et même leurs enfants les suivent...

\section{Conclusion}

Cet accord du collectif au féminin singulier rappelle l'une des valeurs primitives du suffixe $(-a(t)$ ) comme marque de collectif des groupes humains en sémitique. Dans les dialectes arabes que nous avons étudiés, elle est devenue marque de pluriel pour les noms de métier et les ethnonymes (cf. I.C.i.6). 
Sans doute conviendrait-il de pousser plus loin la comparaison car on note, en effet, que toutes les formes dites "féminines", qu'elles soient verbales, pronominales, adjectivales ou participiales, sont souvent liées à un pluriel : pluriels inanimés comme en arabe classique et dans un niveau "médian" pour les dialectes, pluriels humains dans beaucoup de dialectes, pluriels animés et inanimés à Malte.

Une étude plus approfondie de ce lien entre le nombre et le féminin singulier, entre la recatégorisation et l'opposition de genre reste à faire.

\section{BIBLIOGRAPHIE}

- Pour les dialectes yéménites (du Nord) :

P. Behnstedt : Die Nordjemenitischen Dialekte. Teil I : Atlas, Wiesbaden, 1985.

Die Dialekte der Gegend von Saedah (Nord-Jemen), Wiesbaden, 1987.

W. Diem : Skizzen Jemenitischer Dialekte, Beyrouth, 1973.

E. Rossi : L'arabo parlato a San $\bar{\sigma}^{2}$. Grammatica, Testi, Lessico, Roma, 1939.

- Pour les dialectes tunisiens :

G. Boris : Documents linguistiques et ethnographiques sur une région du Sud-tunisien (Nefzaoua), Paris, 1951.

Lexique du parler arabe des Marazig, Paris, 1958.

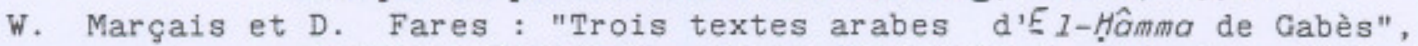
Journal Asiatique, Avril-Juin 1931 ; Oct.-Déc. 1932 ; Juil.-Sept. 1933.

W. Marçais et A. Guíga : Textes arabes de Takroûna (textes et glossaire), Paris, 1925 et 1955-61.

M.-C. Simeone-Senelle: Les formes verbales "dérivées" dans des dialectes maghrébins - Etude morphologique, sémantique et lexicale. Thèse de 3 ème cycle. Paris III. 1984.

M. Darot : Problèmes de détermination et de quantification en arabe dialectal tunisien, Thèse de zème cycle, Paris 7, 1981 .

- Pour le dialecte hassānîya :

D. Cohen : Le dialecte arabe hassānīya de Mauritanie, Paris, 1963.

- Pour le dialecte égyptien :

M. Hinds Arahice Beyront rie du Liban. 1986.

A. M. Sallam : "Concordial relations within the noun-phrase in Educated Spoken Arabic (ESA)", in Archivum Linguisticum, $X, 1$ (new series), 1979.

- Pour le dialecte syrien (Damas) :

M. W. Cowell : A reference grammar of Syrian Arabic (based on the dialect of Damascus), Georgetown University Press, Washington D.C., 1964 . 
- Pour le dialecte marocain (1a région de Fès) :

Dominique Caubet : Grammalre d'uncvarleté d'arabe marocain (région de Fès), Thèse d'état, Paris 7, 1989.

- Pour Malte :

J. Aquilina: The Structure of Maltese. Malte : The Royal University of Malta. 1973 (1959).

Maltese-English Dictionary. Volume One A-L. Malte: Midsea Books. 1987.

L. Schembri : Damma ta' Kliem. Dizzjunarju Malti-Ingliż-Taljan. Malte : P.E.G. 1986.

E.F. Sutcliffe : A Grammar of the Maltese Language. Malte : Progress Press. 196ø (1936).

- Pour le Liban :

M. Feghali : Syntaxe des parlers arabes actuels du Liban. Paris : Imprimerie Nationale. 1928.

- Ouvrages généraux :

D. Cohen : Séminaires donnés à l'E.P.H.E. "Morphogènèse et morphosynthèse en sémitique". 1987-1988.

M. Feghali et A. Cuny : Du genre grammatical en sémitique. Paris: Paul Geuthner. 1924.

H. Fleish : Traité de philologie arabe. Vol. I : Préliminaires. Phonetique. Morphologie nominale. Beyrouth : Implimerie Catholiquxe (Recherches publiégs sous la djrection de l'Institut de lettres orientales de Bełrouth/Tome XVI). 1961 .

W. Marçais : "Le nom d'une fois dans le parler arabe de Djendouba (Nord-ouest tunisien)". Cinquantenalre de l'Ecole Pratique des Hautes Etudes. Paris $f$ Ghampion. 1921.

Les autres données sont celles recueillies par les trois auteurs lors de leurs enquêtes sur le terrain.

N.B. La transcription des termes arabes a été harmonisêe, c'est celle adoptée traditionnellement par les arabisants. 